\title{
Editorial: Wired for Life
}

\author{
Amelia-Elena Rotaru ${ }^{1 *}$ and Pravin M. Shrestha ${ }^{2}$ \\ ${ }^{1}$ Department of Biology, Nordic Center for Earth Evolution, University of Southern Denmark, Odense, Denmark, ${ }^{2}$ Energy \\ Biosciences Institute, University of California, Berkeley, Berkeley, CA, USA
}

Keywords: extracellular electron transfer, electrogens, electrotrophs, iron-reducers

The Editorial on the Research Topic

Wired for Life

Fossil fuels and fossil fuel derived chemicals are limited, non-renewable, and foreseen to run out in the near future. To find viable solutions for the production of renewable fuels and chemicals, significant efforts are being made by the scientific community. For example, some microorganisms have been shown to produce fuels or chemicals using renewable electricity charged electrodes as electron donors for their metabolism (Tremblay and Zhang). The ability to utilize insoluble substrates such as electrodes is due to the property of certain microorganisms to release or take up electrons from insoluble substrates, a process known as extracellular electron transfer (EET).

During EET to a solid electron acceptor, cells dispose of excess electrons indirectly by exploiting shuttles, or directly via a network of conductive pili and cytochromes (Lovley et al., 2011). The same EET mechanisms are considered to assist microorganisms when taking up electrons from solid

OPEN ACCESS

Edited by:

Biswarup Mukhopadhyay,

Virginia Polytechnic Institute and State

University, USA

Reviewed by:

Thomas E. Hanson,

University of Delaware, USA

Husen Zhang,

National Institutes of Health, USA

*Correspondence:

Amelia-Elena Rotaru

arotaru@biology.sdu.dk

Specialty section:

This article was submitted to

Microbial Physiology and Metabolism,

a section of the journal

Frontiers in Microbiology

Received: 18 February 2016

Accepted: 21 April 2016

Published: 10 May 2016

Citation:

Rotaru A-E and Shrestha PM (2016) Editorial: Wired for Life.

Front. Microbiol. 7:662.

doi: 10.3389/fmich.2016.00662 electron donors, however less is known about this process (Tremblay and Zhang). Microorganisms also use direct EET for syntrophic interspecies interactions between a donor species and an acceptor species (Kouzuma et al.; Shrestha and Rotaru).

The papers in this research topic present insights into EET and how this process governs microbe-microbe, microbe-mineral, and microbe-electrodes interactions.

\section{INTERACTIONS BETWEEN MICROBES AND ELECTRODES OR MINERALS}

\section{Isolation of Novel Bacteria Capable of EET}

In this research topic, three independent studies used unusual strategies to enrich or isolate novel bacteria capable of EET.

One study used an electrochemical strategy to isolate electrode and mineral-oxidizing bacteria (Rowe et al.). In this study, Rowe et al., used a three-step approach to enrich electro-active microorganisms from Catalina harbor sediments. Ultimately using solid substrates, like $\mathrm{Fe}^{0}, \mathrm{FeS}$, and $S^{0}, 16$ new species were isolated. All isolates were electroactive and related to six different genera, two of which were for the first time shown to include microorganisms capable of EET.

$\mathrm{Li}$ and Nealson studied microorganisms from marine sediments, which were able to use electrodes as electron acceptors. It is well known that sulfide accumulation inhibits the growth of many microorganisms, however conveniently sulfide can be removed electrochemically (Rabaey et al., 2006; Gong et al., 2013). By investigating electrode-associated microbial communities before and after electrochemical sulfide oxidation Li and Nealson were able to demonstrate a shift from primarily sulfate reducing species to a Clostridia- and Arcobacter-dominated system.

Novel EET microorganisms were also isolated by Hori et al. using iron-hydrooxide minerals as electron acceptors. Six dissimilar $\mathrm{Fe}^{3+}$-reducers were isolated from different environments using acetate as electron donor. As a result, Hori et al. added 
five additional species to the known mineral-reducing genus Geobacter and one to Pelobacter.

\section{Real-Time Monitoring of Microbial Activity}

Typically, when electrodes are used as terminal electron acceptor, microorganisms build biofilms on its solid surface. One paper in this research topic deals with real-time analyses of such electrodeassociated biofilms. Aracic et al. highlighted real time geneexpression profiling, as method of choice, to assess the activity of electroactive biofilms.

Another paper by Wardman et al. showed how microbial activity can be monitored in real time in aquatic sediments using a simple anode-resistor-cathode device. Current production rates correlated with rates of anaerobic microbial activity more specifically with $2-{ }^{14} \mathrm{C}$-acetate turnover. The authors suggested to use this simple sensor technology for in situ monitoring of microbial activity during organic decontamination in sediments.

\section{INTERSPECIES INTERACTIONS}

EET is also important for syntrophic interactions in natural or manmade methanogenic environments. Usually, syntrophic interactions are based on transfer of diffusible chemicals like $\mathrm{H}_{2}$ from a bacterium to a methanogenic archaeon. In a review, Kouzuma et al. highlighted, most recent discoveries in cooperative interactions including interactions via biotic and abiotic electrical conduits.

Many methanogenic archaea involved in syntrophy are hydrogenotrophs, scavenging excess reducing equivalents released, as $\mathrm{H}_{2}$, by their partner bacteria. However, methanogens from the family Methanosarcinales are also acetotrophs, and some feed strictly on acetate. During growth on acetate, Methanosarcina species produce $\mathrm{H}_{2}$ (Lovley and Ferry, 1985), which is cycled back into the cells and reoxidized, with consecutive transfer of electrons and generation of proton motive force. $\mathrm{H}_{2}$ cycling is required for energy metabolism in Methanosarcina (Kulkarni et al., 2009). However, the $\mathrm{H}_{2}$ produced by Methanosarcina can be scavenged by hydrogenotrophic sulfate reducers (Phelps et al., 1985), parasitizing a key energy source for the methanogen. Here, Ozoulmes et al. show that $\mathrm{H}_{2}$ produced by a strict acetoclastic methanogen, Methanosaeta concilii, could also sustain the growth of sulfate reducers or hydrogenotrophic methanogens.

Zheng et al. observed that Methanosarcina spp. co-existed with Geobacter spp. in enrichments from iron-rich sediments of the Jiehe River provided with acetate. The authors suggested that Geobacter and Methanosarcina mazei perform syntrophic acetate oxidation in Jiehe's riverine sediments, which is possibly mediated by naturally abundant conductive minerals, such as magnetite.

Rotaru et al. reported on direct interspecies electron transfer (DIET) between Methanosarcina barkeri and different Geobacter species. The authors, learned that only high current density producing Geobacter species could interact via DIET syntrophy with $M$. barkeri (Rotaru et al.), whereas low current density producers did not. The authors suggested that greatest electrogens evolved effective EET with the purpose to interact syntrophically via DIET with other microorganisms, rather than minerals.

Moreover, a quinone-mediated interaction between Geobacter species (Liu et al., 2012) is described in depth by Smith et al. Quinone moieties are building blocks of humic acidsabundant constituents in soils and sediments. Smith et al. showed that a humic acid analog, AQDS, mediated electron transfer between two Geobacter species. When Geobacter-cocultures were amended with AQDS, they did not need pili and cytochromes, components essential for DIET (Shrestha et al., 2013; Rotaru et al., 2014a,b).

\section{MECHANISMS OF EXTRACELLULAR ELECTRON TRANSFER}

The mechanisms employed by bacteria operating via EET are poorly understood. The best studied are Geobacter sp. and Shewanella sp. In Geobacter species, cell surface components (pili and type $\mathrm{c}$ cytochromes) are required for the organism to be able to grow using insoluble substrates (Lovley et al., 2011). However, in Shewanella, EET occurs via microbial exvaginations of cell membranes which carry redox-active molecules, e.g., multiheme cytochromes, responsible for charge transfer (Pirbadian et al., 2014).

Three papers in this topic studied EET in different organisms thereby expanding our understanding of EET mechanisms (Beckwith et al.; Dalla Vecchia et al.; Shi et al.).

Shi et al. screened all microbial genomes available, and learned that all Geobacter species and 11 phylogenetically and functionally diverse bacteria encoded a protein complex (Pcc) involved in trans-outermembrane electron transfer. The $11 \mathrm{new}$ species spanned physiologies from $\mathrm{Fe}^{3+}$-reducers to anaerobic ammonium oxidizers. The authors suggested that the presence of the Pcc-cluster is an indication these organisms could use extracellular electron acceptors.

The study by Beckwith et al. investigated EET in S. lithotrophicus, an organism capable of $\mathrm{Fe}^{2+}$ oxidation. S. lithotrophicus employs membrane-bound cytochromes for electron uptake from $\mathrm{Fe}^{2+}$. The membrane-bound cytochromes of S. litotrophicus are encoded by $m t o A B$, resembling MtrA and MtrB from Shewanella. The exact mechanism of EET in S. lithotrophicus is unclear. Beckwith et al. anticipate that MtoD, a third c-type cytochrome co-transcribed with MtoAB, plays a role in EET. The authors provided a thorough biochemical and biophysical description of $\mathrm{MtoD}$, and suggested that it plays a role in shuttling electrons between the outer-membrane bound MtoAB and inner-membrane bound electron acceptors.

Finally, EET mechanisms in Gram-positive organisms were studied in Desulfotomaculum reducens cultivated on insoluble iron-oxides (Dalla Vecchia et al.). EET in D. reducens is thought to be cytochrome-independent. The authors investigated cell surface proteins and discovered that only one redox-active protein, a homolog of alkyl hydroperoxide reductase, was differentially expressed in cells grown on iron oxides compared to cells grown fermentatively (Dalla Vecchia et al.). How this protein contributes to EET is not yet clear. 


\section{APPLICATIONS AND ENGINEERING OF EET}

Two papers in this topic discussed the applicability of electroactive properties of microorganisms for sustainable manufacturing of chemicals. Tremblay and Zhang reviewed the use of electroautotrophic microorganisms as catalysts for the production of valuable chemicals when an electrode is the sole electron donor and $\mathrm{CO}_{2}$ is the only electron acceptor and carbonsource.

The second study revealed a novel bioengineering approach in engineered Pseudomonas putida (Schmitz et al.). The authors heterologously expressed a gene cluster for phenazine synthesis in the non-pathogenic P. putida KT2440, an organism of industrial interest, for the production of biodetergents. Usually, P. putida produced biodetergents under high-oxygen conditions, which prompts severe foaming. $\mathrm{O}_{2}$-limiting conditions can prevent foaming. Now, Schmitz et al. bioengineered P. putida to live under $\mathrm{O}_{2}$ limiting conditions with the electrode as electron acceptor, by using phenazine as EET shuttle. This study illustrated a path to $\mathrm{O}_{2}$-limited biocatalysis in $P$. putida and related organisms.

\section{CONCLUSION}

EET has potential for future applications in sustainable technologies, but first we must learn how it works (Tremblay

\section{REFERENCES}

Gong, Y., Ebrahim, A., Feist, A. M., Embree, M., Zhang, T., Lovley, D., et al. (2013). Sulfide-driven microbial electrosynthesis. Environ. Sci. Technol. 47, 568-573. doi: $10.1021 /$ es303837j

Kulkarni, G., Kridelbaugh, D. M., Guss, A. M., and Metcalf, W. W. (2009). Hydrogen is a preferred intermediate in the energy-conserving electron transport chain of Methanosarcina barkeri. Proc. Natl. Acad. Sci. U.S.A. 106, 15915-15920. doi: 10.1073/pnas.0905914106

Liu, F., Rotaru, A.-E., Shrestha, P. M., Malvankar, N. S., Nevin, K. P., and Lovley, D. R. (2012). Promoting direct interspecies electron transfer with activated carbon. Energy Environ. Sci. 5, 8982. doi: 10.1039/c2ee22459c

Lovley, D. R., and Ferry, J. G. (1985). Production and consumption of $\mathrm{H}_{2}$ during growth of Methanosarcina spp. on acetate. Appl. Environ. Microbiol. 49, 247-249.

Lovley, D. R., Ueki, T., Zhang, T., Malvankar, N. S., Shrestha, P. M., Flanagan, K. A., et al. (2011). Geobacter: the microbe electric's physiology, ecology, and practical applications. Adv. Microb. Physiol. 59, 1-100. doi: 10.1016/b978-0-12-3876614.00004-5

Phelps, T. J., Conrad, R., and Zeikus, J. G. (1985). Sulfate-dependent interspecies H2 transfer between Methanosarcina barkeri and Desulfovibrio vulgaris during coculture metabolism of acetate or methanol. Appl. Environ. Microbiol. 50, 589-594.

Pirbadian, S., Barchinger, S. E., Leung, K. M., Byun, H. S., Jangir, Y., Bouhenni, R. A., et al. (2014). Shewanella oneidensis MR-1 nanowires are outer membrane and periplasmic extensions of the extracellular electron transport components. Proc. Natl. Acad. Sci. U.S.A. 111, 12883-12888. doi: 10.1073/pnas.1410551111 and Zhang). This research topic has collected recent groundbreaking advances in our understanding of what microbes utilize EET, how EET is carried out, and how it may be utilized in engineered systems. We hope that the topic persuades more researchers to explore EET in less investigated microorganisms, which are of interest for future applications.

\section{AUTHOR CONTRIBUTIONS}

All authors listed, have made substantial, direct, and intellectual contribution to the work, and approved it for publication.

\section{FUNDING}

AR would like to acknowledge the funding agencies which provided support for the duration of this research topic, namely two Natur of Univers, Det Frie Forskningsråd grants (an Independent research grant no. 1325-00022A, and a Sapere Aude Research Leadership Award no. 4181-00203), the Novo Nordisk Foundation, and Innovation fond no. 4106-00017B.

\section{ACKNOWLEDGMENTS}

We would like to acknowledge Editor Tom Hanson for kindly reading the manuscript and providing advice.

Rabaey, K., Van de Sompel, K., Maignien, L., Boon, N., Aelterman, P., Clauwaert, P., et al. (2006). Microbial fuel cells for sulfide removal. Environ. Sci. Technol. 40, 5218-5224. doi: 10.1021/es0 $60382 \mathrm{u}$

Rotaru, A.-E., Shrestha, P. M., Liu, F., Shrestha, M., Shrestha, D., Embree, M., et al. (2014a). A new model for electron flow during anaerobic digestion: direct interspecies electron transfer to Methanosaeta for the reduction of carbon dioxide to methane. Energy Environ. Sci. 7, 408-415. doi: 10.1039/ C3EE42189A

Rotaru, A.-E., Shrestha, P. M., Liu, F., Markovaite, B., Chen, S., Nevin, K. P., et al. (2014b). Direct interspecies electron transfer between Geobacter metallireducens and Methanosarcina barkeri. Appl. Environ. Microbiol. 80, 4599-4605. doi: 10.1128/AEM.00895-14

Shrestha, P. M., Rotaru, A.-E., Summers, Z. M., Shrestha, M., Liu, F., and Lovley, D. R. (2013). Transcriptomic and genetic analysis of direct interspecies electron transfer. Appl. Environ. Microbiol. 79, 2397-2404. doi: 10.1128/AEM.03837-12

Conflict of Interest Statement: The authors declare that the research was conducted in the absence of any commercial or financial relationships that could be construed as a potential conflict of interest.

Copyright (C) 2016 Rotaru and Shrestha. This is an open-access article distributed under the terms of the Creative Commons Attribution License (CC BY). The use, distribution or reproduction in other forums is permitted, provided the original author(s) or licensor are credited and that the original publication in this journal is cited, in accordance with accepted academic practice. No use, distribution or reproduction is permitted which does not comply with these terms. 